\title{
Expectativas versus Satisfação dos Consumidores da Rede Hoteleira de Campo Grande-MS
}

\author{
Expectations vis-a-vis Satisfaction of the Users of Hotels in Campo Grande-MS
}

\section{Expectativas versus Satisfacción del Cliente de los Hoteles en Campo Grande-MS}

\author{
Dario de Oliveira Lima-Filho ${ }^{1}$ \\ Izabel Zorzo Marchiotti ${ }^{2}$ \\ Filipe Quevedo-Silva ${ }^{3}$
}

\begin{abstract}
Resumo
O objetivo deste trabalho foi mensurar a satisfação dos consumidores da rede hoteleira de Campo Grande-MS, dentro do paradigma da desconfirmação das expectativas. Para tanto, foi feito um estudo quantitativo-descritivo transversal único junto a 407 indivíduos usuários de hotéis em Campo Grande-MS, de ambos os sexos e idade igual ou superior a 18 anos, em outubro de 2008, usando um questionário estruturado. Foi utilizado o modelo Servqual, por meio de suas cinco dimensões, sendo que na primeira parte foram estimadas as expectativas dos consumidores e na segunda a percepção do serviço prestado pelos hotéis. As variáveis consideradas foram: apresentação das acomodações, área de lazer, restaurante, cortesia dos funcionários, agilidade e eficiência dos funcionários, conhecimento dos funcionários, limpeza do hotel, localização, segurança e atenção personalizada. Os resultados revelam que os hotéis, em geral, não têm satisfeito as expectativas em relação à segurança e aos restaurantes, seguidos pela apresentação das acomodações e conhecimento dos funcionários. Para os hotéis da categoria A, a grande maioria das variáveis apresentou um alto grau de satisfação, para a categoria $\mathrm{B}$ as variáveis de maior valor são segurança, cortesia, agilidade e eficiência dos funcionários, seguidas por acomodações e atenção personalizada. Para a categoria $\mathrm{C}$, os atributos mais importantes são a segurança, a agilidade e eficiência dos funcionários e a cortesia dos mesmos. Já os hotéis da classe D são os que menos proporcionam satisfação ao seu público.
\end{abstract}

Palavras-chave: modelo de Oliver; turismo, estratégia, hotéis.

\footnotetext{
1 Doutor em administração pela FGV-SP. Professor Associado do Programa de Pós-Graduação em Administração da Universidade Federal de Mato Grosso do Sul (UFMS). Coordena diversos projetos de pesquisa financiados pelo CNPq e pela FINEP. E-mail: dariolimafilho@gmail.com.

${ }^{2}$ Graduada em administração pela Universidade Federal de Mato Grosso do Sul. E-mail: bellzm@hotmail.com. ${ }^{3}$ Mestrando em administração pela Universidade Federal de Mato Grosso do Sul. E-mail: admquevedo@hotmail.com.
} 


\begin{abstract}
This work was developed to measure the customers' satisfaction of Campo Grande-MS hotels, under the paradigm of disconfirmation of expectations. For that, was made a conclusively quantitative-descriptive study cross only with with 407 individuals, users of hotels in Campo Grande-MS, of both sexes aged more than 18 years old, in October 2008, using a structured questionnaire. It was used the SERVQUAL method to construct the questionnaire and through its five dimensions were estimated the costumers expectations in the first part and the perception of the service offered by hotels in the second one. The variables considered were the presentation of accommodation, leisure area, restaurant, courtesy of staff, agility and efficiency of staff, knowledge of staff, cleanliness of the hotel, location, safety and personal attention. The results showed that for most consumers the hotels have generally not met your expectations for safety and restaurants, followed by the presentation of the accommodation and knowledge of employees. For hotels of category $A$, the vast majority of variables showed a high degree of satisfaction, for category $B$ the variables more important were safety, courtesy, responsiveness and efficiency of employees, followed by accommodation and personal attention. For category $C$, the most important attributes are safety, agility and efficiency of employees and their courtesy. The D class hotels are the less provide satisfaction to their audiences.
\end{abstract}

Keywords: model of Oliver; tourism; strategy; hotels.

\title{
Resumen
}

El objetivo de este estudio fue medir la satisfacción del cliente de los hoteles en Campo Grande-MS, dentro del paradigma de la desconfirmación de expectativas. A tal fin, se realizó un estudio cuantitativo, transversal único con 407 usuarios de los hoteles en Campo Grande$M S$, de ambos sexos y mayores de 18 años en octubre 2008, utilizando un cuestionario estructurado. Se utilizó el modelo SERVQUAL, a través de sus cinco dimensiones, en la primer parte se estima las expectativas del consumidor y en la segunda parte la percepción del servicio que ofrecen los hoteles. Las variables consideradas fueron: presentación de alojamiento, área de recreación, restaurante, cortesía del personal, la agilidad y la eficiencia de los empleados, los conocimientos del personal, limpieza del hotel, ubicación, seguridad y atención personalizada. Los resultados revelan que los hoteles en general no han cumplido con las expectativas de los restaurantes de seguridad y, seguido por la presentación de los alojamientos y el conocimiento de los empleados. Para los hoteles de categoría A, la gran mayoría de las variables mostraron un alto grado de satisfacción, para la categoría $B$ las variables más importantes son seguridad, la cortesía, la capacidad de respuesta y eficiencia de los empleados, seguido por el alojamiento y la atención personal. Para la categoría $C$, los atributos más importantes son la seguridad, la agilidad y la eficiencia de los empleados y su cortesía. Los hoteles de clase D son los que menos proveen satisfacción a sus audiencias.

Palabras clave: modelo de Oliver; turismo; estrategia; hoteles. 


\section{Introdução}

O setor de turismo, que está inserido na categoria de serviços, inclui o segmento de hotelaria. O setor ocupa hoje papel relevante na economia mundial, sendo uma das atividades com maior representatividade econômica, ao lado da indústria do petróleo. Segundo informações do IBGE (2010), as atividades características do turismo no Brasil tiveram um aumento na sua produção de aproximadamente 39,7\% de 2004 a 2008 e contaram com 5,9 milhões de postos de trabalho em 2007.

Com o desenvolvimento desse setor, a qualidade dos serviços, a satisfação e a fidelidade do cliente ganharam incentivos para estudos acadêmicos mais profundos. A busca por ofertar melhor qualidade nos produtos e serviços e, consequentemente, garantir a satisfação dos clientes, começou a orientar as estratégias das empresas no intuito de consolidar suas posições diante dos concorrentes ou de fazer crescer sua participação no mercado (MACHADO, 2001). Atualmente, as empresas prestadoras de serviços precisam conhecer os consumidores de seus produtos e a maneira como se comportam, pois formulações de estratégias para solidificação no mercado são dependentes desse conhecimento, uma vez que os clientes buscam, cada vez mais, contato com empresas que, além de satisfazerem suas necessidades individuais, os surpreendam e sejam capazes de oferecer bens e serviços baseados em seus desejos específicos; o objetivo deixa é o pleno contentamento dos consumidores para que estes se mantenham leais. Nesse contexto, vale ressaltar que a satisfação do consumidor resulta do sentimento de realização de uma necessidade ou desejo, fruto da comparação dos produtos ou serviços realizados em comparação com as expectativas geradas.

Esse estudo tem como objetivo avaliar a satisfação dos consumidores da rede hoteleira da cidade de Campo Grande, Mato Grosso do Sul. Em termos específicos pretende: a) verificar a expectativa dos entrevistados em relação aos serviços oferecidos pelos hotéis; b) verificar a percepção dos clientes sobre os serviços prestados pelos hotéis; c) identificar o grau de satisfação dos clientes. 


\section{Referencial Teórico}

\subsection{Satisfação}

Os pesquisadores da área concordam com a premissa de que a satisfação no consumo de um bem ou serviço beneficia tanto os consumidores quanto as organizações, porém o conceito de satisfação ainda não é um consenso entre os estudiosos (OLIVER, 1997).

Oliver (1997) estabelece sua definição a partir da origem do vocábulo satisfação. O termo deriva do latim nos seus dois componentes: o anteposto satis (bastante, suficiente) e o pospositivo fação/facção (maneira de fazer ou poder de fazer). Entende-se, a partir daí, que produtos ou serviços que produzem satisfação são aqueles que têm a capacidade de fornecer o que se está procurando até o ponto de ser bastante ou suficiente.

Para a maioria dos autores o grau de satisfação decorre de uma comparação efetuada pelo consumidor, comparação esta que confronta o resultado da transação com uma referência anteriormente existente (SPRENG, MACKENZIE; OLSHAVSKY, 1996). Para Oliver (1997), expectativas, necessidades, qualidade, iniqüidade e arrependimento constituem padrões comparativos, que atuam em paralelo.

A satisfação, uma vez definida como um estado psicológico, não pode ser analisada diretamente (EVRARD, 1994). No entanto, devido à necessidade acadêmica e prática da mensuração da satisfação, tem-se procurado desenvolver escalas para medi-la.

Os resultados dessas pesquisas desencadearam diversos estudos a partir da década de 1970, com o objetivo de desvendar os antecedentes da satisfação do consumidor e de desenvolver medidas confiáveis do construto. Grande parte deles foi baseada no paradigma da desconfirmação, de acordo com o qual a satisfação está relacionada ao tamanho e à direção da desconfirmação; esta se relaciona às expectativas do consumidor anteriores à experiência de consumo (CHURCHILL; SURPRENANT, 1982). Os estudos realizados sobre o tema permitem afirmar que as expectativas assumiram maior relação com a satisfação que todas as variáveis demais, como idade, escolaridade ou classe social. Assim sendo, a teoria do paradigma da desconfirmação será utilizada nesse trabalho como sendo o fator relevante na predição da satisfação do consumidor. 
Conclusões de diversos estudos sobre os preditores da satisfação do consumidor até 1990 foram consolidados por Yi (1990), que encontrou resultados controversos sobre o poder preditivo de variáveis demográficas e sócio-psicológicas. Por um lado, a literatura apontava para resultados como o aumento da satisfação do consumidor com a idade e com a competência pessoal e o seu decréscimo com a educação e a renda familiar, no entanto, apresentava também evidências de relações pouco ou não significativas entre essas variáveis.

Logo, o principal foco de estudo a respeito dos preditores da satisfação foi sobre a avaliação pós-consumo do desempenho do produto relacionado a processos cognitivos como a desconfirmação (Yi, 1990). Churchill e Surprenant (1982) consideram os estudos de Cardozo (1965), Olshavsky e Miller (1972) e Anderson (1973) como o fundamento sobre o qual foram realizados os estudos posteriores na área da satisfação do consumidor. Nos estudos mencionados, foram investigados os efeitos das expectativas sobre a satisfação (CARDOZO, 1965), assim como o papel da desconfirmação de expectativas sobre a percepção de desempenho do produto (OLSHAVSK; MILLER, 1972; ANDERSON, 1973).

A recomendação de Oliver (1997), no que tange à mensuração da satisfação do consumidor, é a de que o instrumento de pesquisa seja construído de forma que cada dimensão (ou atributo) seja medida em termos de expectativas, desconfirmação e desempenho, a fim de identificar os preditores psicológicos mais fortes da satisfação no caso de cada dimensão do serviço. Segundo ele, os resultados poderiam revelar preditores da satisfação baseados em desconfirmação, expectativas ou desempenho percebido. Nesse trabalho, será o utilizado o modelo de Parasuraman et al. (1988).

A maioria dos estudos sobre o assunto concorda que a satisfação é resultado de um processo de comparação. Para poder esclarecer a afirmativa, alguns modelos têm sido sugeridos (EVRARD, 1994). Em uma revisão da literatura no intuito de integrar os esforços de pesquisa de 1977 até 1990, Tse et al. (1990) identificaram dois paradigmas que orientaram os estudos sobre a satisfação do consumidor. O primeiro enfoca as interações pós-consumo que funcionam como restauradoras do desequilíbrio psicológico que se segue à experiência de consumo. O segundo, de maior relevância para os estudos sobre expectativas e satisfação do consumidor, é o do Paradigma da Desconfirmação, que será abordado mais profundamente. 
Oliver (1997) designa este termo à teoria que adota como referência as expectativas, uma vez que, como os primeiros estudos sobre a satisfação foram realizados utilizando a expectativa preditiva como padrão, o termo desconfirmação de expectativas passou a referir-se a este conceito. Esse paradigma tem por componente a relação entre expectativas e performance, que origina a desconfirmação de expectativas. Esta, portanto, é uma das principais responsáveis pela indicação da satisfação do consumidor e da qualidade percebida pelo modelo dos GAPS (PARASURAMAN; ZEITHAML; BERRY, 1994). Para Parasuraman, Zeithaml e Berry (1994), qualidade percebida é a opinião do consumidor sobre a excelência ou superioridade de uma organização como um todo. É uma forma de atitude, relacionada (mas não equivalente) à satisfação e que resulta de uma comparação de expectativas com percepções da performance.

Oliver (1980) descreve a teoria da desconfirmação da seguinte forma: as expectativas marcam um ponto de referência a partir do qual se faz um julgamento comparativo. Dessa forma, pode haver uma desconfirmação negativa, quando o julgamento é inferior ao ponto de referência, ou desconfirmação positiva, quando os resultados são melhores do que o esperado. O modelo prevê, portanto, que a satisfação ocorrerá quando o consumidor comparar a sua própria percepção de desempenho com a expectativa que tinha do mesmo (SPRENG; MACKENZIE; OLSHAVSKY, 1996).

Existem dois níveis de serviço: o desejado e o adequado. O primeiro reflete o serviço que o cliente espera receber; o segundo, o que o cliente considera aceitável. Separando estes dois níveis de serviço, existe uma área denominada "zona de tolerância”, que é o âmbito do desempenho do serviço considerado satisfatório pelo cliente. Um desempenho abaixo dessa zona gera nele frustração e reduz sua lealdade, enquanto que um desempenho acima dela o surpreenderá agradavelmente, reforçando sua fidelidade (BERRY; PARASURAMAN, 1995). Enquanto a satisfação estaria relacionada aos processos de desconfirmação zero e positiva, a insatisfação seria resultado da desconfirmação negativa das expectativas do consumidor. (CHURCHILL; SURPRENANT, 1982).

O conhecimento acumulado sobre a desconfirmação e a satisfação levou a três possibilidades para demonstrar o relacionamento existente entre os dois conceitos, os quais podem ser visualizados na Figura 1. 

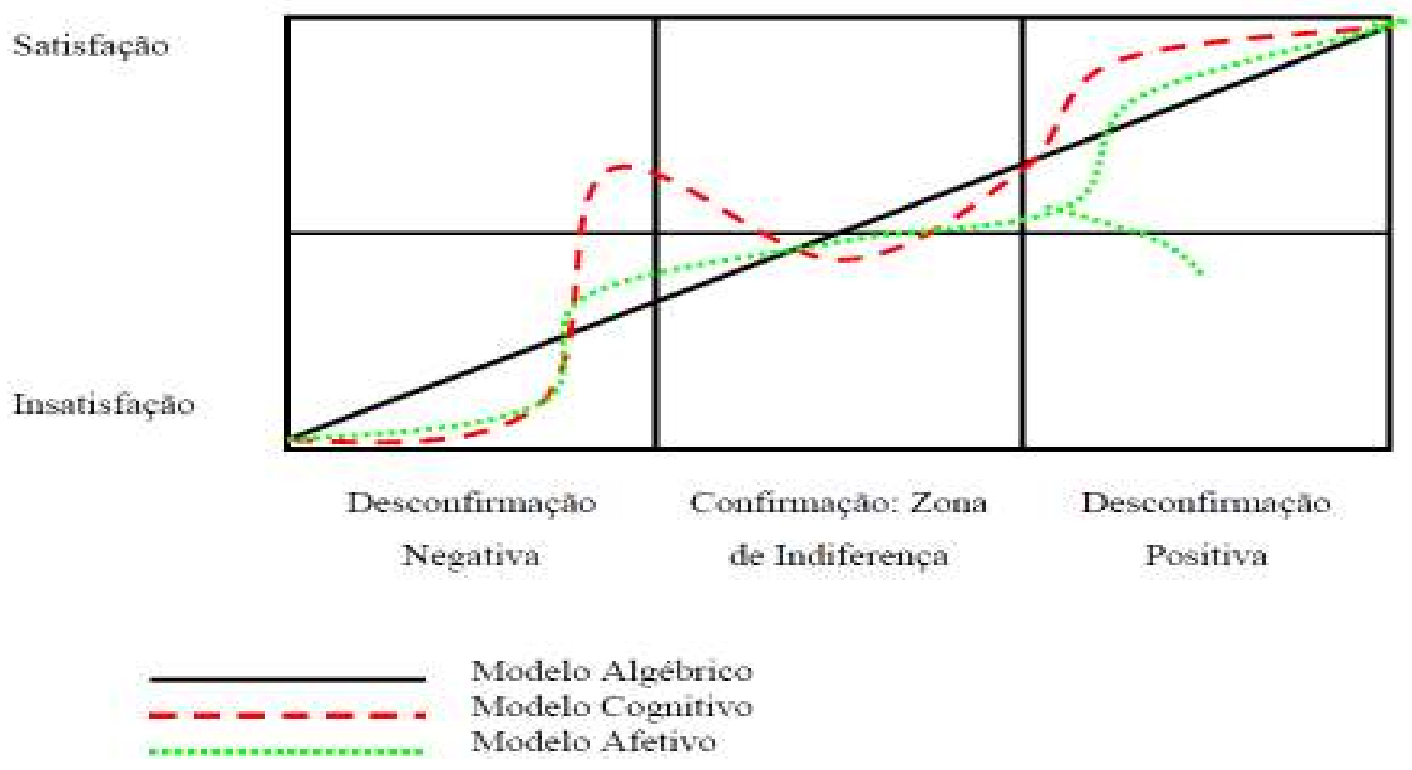

Figura 1 - A relação entre desconfirmação e satisfação

Fonte: Evrard (1994)

Conforme observam Spreng, MacKenzie e Olshavsky (1996), não há na literatura um consenso claro acerca do conceito de expectativas. Estes autores preferem reservar o termo à percepção de probabilidade de ocorrência de determinado evento. Outros, como Oliver (1980), defendem que o conceito deve ser ampliado e incorporar a avaliação, positiva, negativa ou neutra, do evento esperado. Independentemente dessas diferenças, as duas tendências reconhecem que a expectativa preditiva (isto é, restrita à percepção de probabilidade de ocorrência do evento) é insuficiente para dar conta do fenômeno da satisfação. A maior parte dos autores (EVRARD, 1994; OLIVER, 1997; SPRENG, MACKENZIE; OLSHAVSKY, 1996, entre outros) admite que as expectativas sejam apenas um dos elementos do quadro de referência a partir do qual os consumidores avaliam suas experiências. Spreng, MacKenzie e Oshlavsky (1996) argumentam que os desejos (expectativas) devem ser incorporados ao modelo proposto pelo paradigma da desconfirmação.

Devido ao acúmulo de forte sustentação empírica, atualmente esse é o modelo teórico dominante em diversos campos de estudo da satisfação, embora diversos elementos do modelo ainda necessitem de refinamento e testes (OLIVER, 1997). O paradigma da 
desconfirmação articula expectativas, percepção de desempenho do produto, desconfirmação e satisfação (CHURCHILL; SURPRENANT, 1982). Por esse motivo, o paradigma da desconfirmação de expectativas foi eleito como quadro de referência para a presente pesquisa.

\subsection{Indústria hoteleira}

O negócio de hospedagem é quase tão antigo quanto a própria civilização. Segundo Ismail (2004), a história da hospedagem data desde os tempos das civilizações da Suméria e do antigo Egito, pois a necessidade de dispor de um local de repouso quando longe de casa é tão antiga quanto o primeiro viajante nômade.

A partir da II Guerra Mundial, o turismo passou a ter grande repercussão e tornou-se uma atividade econômica bastante significativa, a qual pode ser considerada a grande contribuinte para o crescimento das redes hoteleiras. Conforme aponta Prosérpio (2007), as cadeias de hotéis juntamente com o turismo de massa surgiram de modo significativo por volta de 1950, nos Estados Unidos, e logo se expandiram por todo continente Europeu. Com o passar dos anos, expandiram-se por todo o mundo.

Segundo Carvalho et al (2009), atualmente nota-se uma movimentação no setor hoteleiro em função principalmente dos efeitos da globalização, a internacionalização das empresas, o acirramento da competitividade, os custos mais acessíveis das passagens, facilitando a locomoção das pessoas e outros fatores relacionados.

Segundo Associação Brasileira da Indústria de Hotéis (ABIH, 2008), a indústria hoteleira brasileira gera mais de um milhão de empregos diretos e indiretos e em 2008 obteve uma receita bruta de US\$ 9 bilhões, tendo uma arrecadação de mais de US\$ 1,8 bilhão em impostos e taxas. Entretanto, de acordo com Pereira (2009) a crescente oferta de oportunidades no setor vem acompanhada de rigorosa exigência de qualidade e produtividade, o que impõe à indústria de hospitalidade brasileira a necessidade de maior profissionalização.

Manter interação com os clientes, funcionários preparados, processos ágeis e flexíveis, bem como qualidade nos serviços, possibilita à indústria hoteleira grande vantagem competitiva e maior produtividade. Anderson e Mittal (2000), estudando empresas que fazem parte do Barômetro de Satisfação do Consumidor Sueco, encontraram um aumento de 1\% no índice de satisfação do consumidor associado ao aumento de $2,37 \%$ no retorno sobre o investimento. Já 
um decréscimo de 1\% neste índice estava associado a 5,08\% de decréscimo no retorno sobre o investimento. Assim, constata-se que não só o aumento da satisfação do consumidor é importante para as empresas, mas também é de extrema relevância evitar que ela diminua.

Em outro estudo realizado na cidade de Natal-RN com o intuito de desvendar os principais atributos de satisfação do consumidor de hotelaria, Gonzáles, Ramos e Amorim (2005) encontraram quatro fatores que se revelaram importantes para a satisfação dos hóspedes: qualidade do quarto de hotel, cordialidade dos funcionários, qualidade do restaurante e a percepção de custo-benefício. Além disso, os autores identificaram que esses quatro fatores são responsáveis por $56 \%$ da satisfação dos hóspedes com o hotel.

Em uma pesquisa realizada no Brasil, Branco, Ribeiro e Tinoco (2010) identificaram atributos de satisfação do consumidor para o serviço de hotelaria. Os autores encontraram seis atributos de satisfação para serviços de hotelaria, apresentados na Tabela 1. Entre esses seis atributos identificados, o que teve maior importância para os hóspedes entrevistados foi o quarto.

Tabela 1 - Atributos de satisfação para os consumidores de hotel

\begin{tabular}{|c|c|c|}
\hline \multicolumn{2}{|c|}{ Atributos } & \multirow{2}{*}{$\begin{array}{l}\text { Contribuição para a } \\
\text { qualidade percebida } \\
27,4 \%\end{array}$} \\
\hline 1) & Quarto & \\
\hline 2) & Atendimento & $20,4 \%$ \\
\hline 3) & Infraestrutura & $16,2 \%$ \\
\hline 4) & Aspectos gerais internos & $13,7 \%$ \\
\hline 5) & Serviços de alimentação & $11,4 \%$ \\
\hline 6) & Aspectos gerais externos & $10,8 \%$ \\
\hline
\end{tabular}

Fonte: Adaptado de Branco, Ribeiro e Tinoco (2010, p. 585)

Os resultados do Market Metrix Hospitality Index (uma das maiores medidas de desempenho percebido da indústria hoteleira americana) revelam que as emoções experimentadas pelos hóspedes exercem uma importante influência sobre as respostas de satisfação e lealdade (BARSKY; NASH, 2003). Estes autores também segmentaram a base de dados do Market Metrix Hospitality Index em vinte e dois grupos distintos de consumidores com base em 
variáveis geodemográficas, comportamentais e atitudinais. Uma análise geográfica da satisfação dos hóspedes revelou diferenças significativas entre regiões, sendo que as cidades com mais baixos índices de satisfação dos hóspedes também eram aquelas com os menores tempos médios de estada, além de apresentarem hóspedes com a maior resistência a preço. Também foram encontradas variações relacionadas a fatores climáticos e às cidades de origem dos hóspedes.

\section{Metodologia}

Foi realizado um estudo quantitativo-descritivo transversal único (MALHOTA, 2001). A técnica utilizada para a coleta de dados foi a entrevista pessoal, sendo que os dados foram coletados nos próprios hotéis em dois momentos: antes dos usuários realizarem check-in no estabelecimento e, outra vez, após o check-out. A amostra é caracterizada como intencional por terem sido selecionados determinados estabelecimentos, não aleatoriamente, nos quais os entrevistadores aplicaram os questionários aos hóspedes.

Para a seleção dos hóspedes entrevistados, foi feita uma amostra aleatória com confiança de 95\% e erro amostral 5\%. Dessa forma, foram entrevistados 407 usuários de hotéis em Campo Grande-MS, de ambos os sexos e idade igual ou superior a 18 anos, nos meses de outubro e novembro de 2008 .

Para a realização dessa pesquisa foram selecionados 26 hotéis, sendo dois deles pertencentes à classe $\mathrm{A}$, seis à classe $\mathrm{B}$, quatro à classe $\mathrm{C}$ e quatorze à classe $\mathrm{D}$, sendo que a classificação utilizada, em quatro tipos, de $\mathrm{A}$ à $\mathrm{D}$, foi definida por dois especialistas na área de hotel de Campo Grande, pois os hotéis do município não se enquadram perfeitamente na classificação oficial da EMBRATUR.

Foi adotado o método SERVQUAL (PARASURAMAN; ZEITHAML; BERRY, 1994) para a avaliação da satisfação dos consumidores, visando captar critérios para avaliar a satisfação dos clientes em relação aos serviços oferecidos. Este modelo, baseado em cinco colunas (gaps), avalia a discrepância entre a expectativa do cliente em relação ao serviço e a percepção do desempenho. Neste trabalho foi priorizada a Gap 5 - lacuna entre o serviço prestado e o serviço percebido, sendo a resultante final em função dos outros quatro gaps. 
O questionário utilizado foi composto por dois blocos de perguntas fechadas. Cada bloco continha 10 variáveis referentes às dimensões propostas pelo padrão SERVQUAL, sendo que o primeiro grupo agregou aquelas que dizem respeito às expectativas, e o segundo as percepções dos clientes. Para a mensuração das variáveis, utilizou-se uma escala Likert de cinco pontos. A tabela 2 mostra as dimensões e variáveis pesquisadas.

Tabela 2 - Dimensões e variáveis analisadas na pesquisa de satisfação do consumidor da rede hoteleira de CG - MS

\begin{tabular}{l|l}
\hline Dimensões mensuradas & Variáveis \\
\hline Tangibilidade & Apresentação das acomodações; \\
& Área de lazer; \\
& Restaurante; \\
& Limpeza; \\
& Localização. \\
\hline Confiabilidade & Agilidade e eficiência dos serviços; \\
& $\begin{array}{l}\text { Conhecimento dos funcionários sobre } \\
\text { produtos e serviços. }\end{array}$ \\
\hline Atendimento & Cortesia e profissionalismo dos atendentes. \\
\hline Segurança & Serviços e equipamentos de segurança. \\
\hline Empatia & Atenção personalizada oferecida. \\
\hline Fonte: Adaptado de Parasuraman et al (1994) &
\end{tabular}

Fonte: Adaptado de Parasuraman et al. (1994)

Para a análise dos resultados foram utilizadas matrizes de atributos, conforme proposto por Albrecht e Bradford (1992). Essas matrizes relacionam a importância atribuída pelos consumidores a um atributo e o nível de desempenho do mesmo. 


\section{Resultados e Discussão}

\subsection{Perfil da amostra}

A Tabela 3 traz as informações sócio-demográficas da amostra estudada.

Tabela 3 - Perfil sociodemográfico dos entrevistados

\begin{tabular}{|c|c|c|c|}
\hline Variáveis & Grupos & Freqüência & Percentual \\
\hline \multirow[t]{2}{*}{ Sexo } & Masculino & 313 & $76.9 \%$ \\
\hline & Feminino & 94 & $23.1 \%$ \\
\hline \multirow[t]{5}{*}{ Idade } & 18 a 24 anos & 51 & $12.5 \%$ \\
\hline & 25 a 34 anos & 130 & $31.9 \%$ \\
\hline & 35 a 44 anos & 114 & $28.0 \%$ \\
\hline & 45 a 54 anos & 77 & $18.9 \%$ \\
\hline & A partir de 55 anos & 35 & $8.6 \%$ \\
\hline \multirow[t]{5}{*}{ Escolaridade } & Analfabeto & 3 & $0.7 \%$ \\
\hline & Fundamental & 51 & $12.5 \%$ \\
\hline & Ensino Médio & 127 & $31.2 \%$ \\
\hline & Superior & 157 & $38.6 \%$ \\
\hline & Pós-graduado & 69 & $17.0 \%$ \\
\hline \multirow[t]{4}{*}{ Classe social } & Classe A & 106 & $26.0 \%$ \\
\hline & Classe B & 201 & $49.4 \%$ \\
\hline & Classe C & 90 & $22.1 \%$ \\
\hline & Classe D & 10 & $2.5 \%$ \\
\hline
\end{tabular}

$\mathrm{N}=386$

Fonte: Elaborado pelos autores com dados de fonte primária.

\subsection{A matriz de atributos}

A matriz de atributos revela o grau de importância atribuído pelos consumidores às principais variáveis identificadas nos hotéis, relacionando esse valor ao desempenho obtido. Além de proporcionar aos hotéis recursos que possibilitam a adoção de posicionamentos compatíveis com os desejos do público, a matriz permite uma avaliação detalhada sobre os atributos que 
devem ser priorizados e aqueles que não agregam grande valor. Os critérios finais de serviço indicam quais atributos terão prioridade para a empresa, ou seja, aqueles que ela deve assumir como mais importantes e sobre os quais deve promover mudanças positivas (ALBRECHT; BRADFORD, 1992).

Os quatro quadrantes da matriz apresentam atributos classificados como tendo: 1) Vulnerabilidade competitiva; 2) Força competitiva; 3) Relativa indiferença; 4) Superioridade irrelevante. O primeiro quadrante representa as variáveis com alta importância e baixa satisfação, nele estão concentrados os atributos que merecem atenção especial da empresa, para que sejam melhorados e atinjam níveis de satisfação mais altos. Na segunda área estão localizadas as variáveis que foram consideradas muito importantes e obtiveram um grau de desempenho alto, ou seja, os clientes valorizam esses serviços e estão satisfeitos com a performance da empresa em relação a eles. No terceiro quadrante estão atributos com desempenho ruim e que são irrelevantes para o consumidor. As organizações, no entanto, não devem deixá-los abandonados, pois apesar de apresentarem pouca importância, isso não significa que eles sejam totalmente desvalorizados, mas que outros serviços são mais estimados do que estes. Por fim, o quadrante de superioridade irrelevante é constituído por atributos sobre os quais os clientes estão satisfeitos, mas cujas médias de importância ficaram abaixo da média geral. Isto é, são serviços que satisfazem os consumidores, mas não geram uma vantagem competitiva significante (ALBRECHT; BRADFORD, 1992).

Além dos quatro quadrantes em que a matriz se divide, há uma área nomeada "zona cinzenta", na qual são agrupados atributos que representam importância e desempenho médios. Essa localização indica que a empresa está no mesmo nível dos concorrentes, não oferecendo diferenciação alguma ao público.

\subsection{Resultados gerais para os hotéis}

Os resultados apontam que os hotéis, de um modo geral, têm satisfeito seus consumidores na maioria dos atributos, conforme demonstrado na Figura 2. 
GERAL

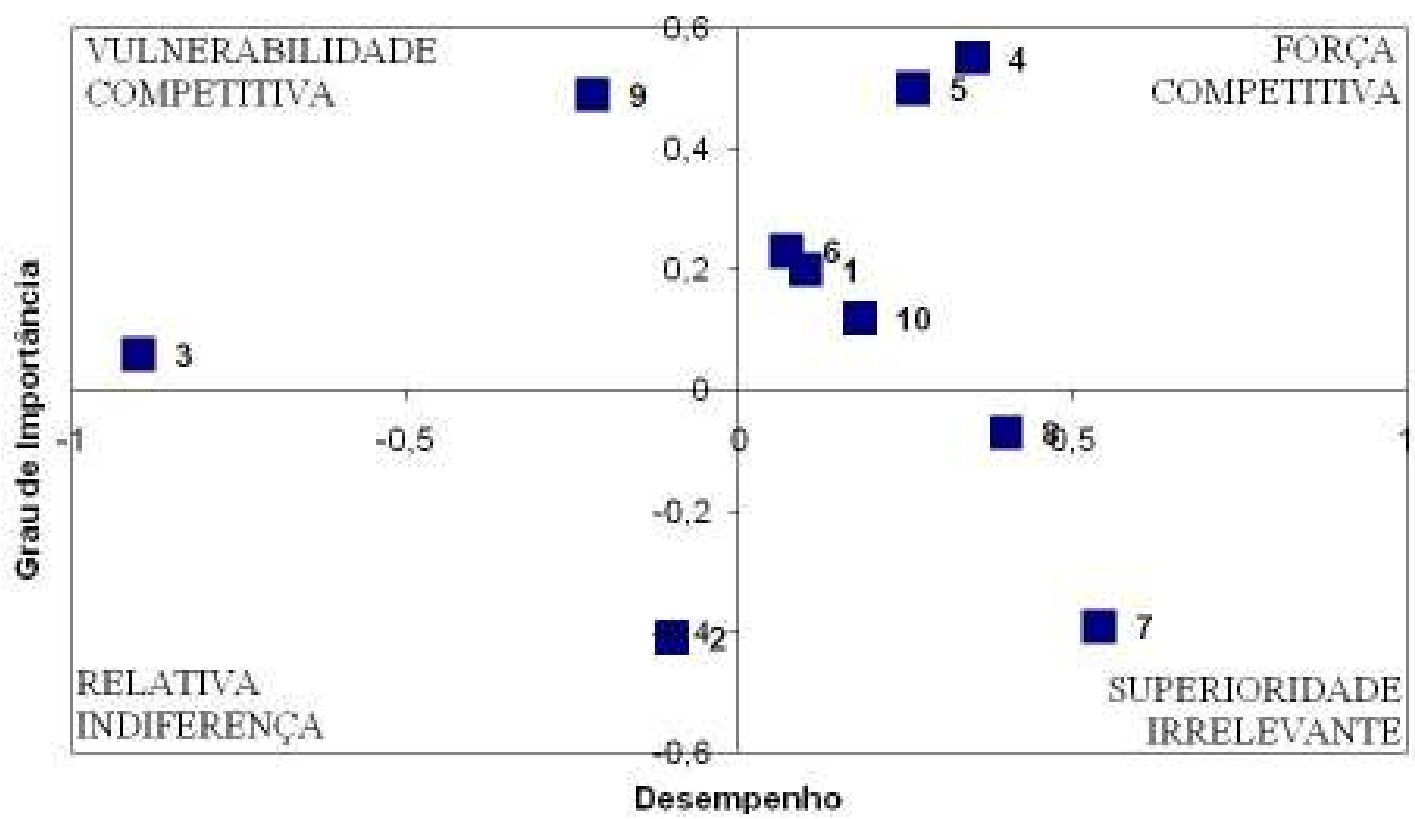

Figura 2 - Matriz de atributos dos hotéis- geral - (1) acomodação; (2) área de lazer; (3) restaurante; (4) cortesia dos funcionários; (5) agilidade e eficiência dos funcionários; (6) conhecimento dos funcionários; (7) limpeza; (8) localização do hotel; (9) segurança; (10) atenção personalizada.

Fonte: Elaborado pelos autores.

Apresentação das acomodações, agilidade e eficiência, cortesia e conhecimento dos funcionários, limpeza, localização e atenção personalizada são variáveis que apresentaram desempenhos acima da média. O enfoque dos hotéis em relação aos atributos localizados no quadrante força competitiva, deve ter o intuito de mantê-los com níveis bons ou excelentes. $\mathrm{O}$ essencial nessa situação é sustentar as vantagens já alcançadas, atentando para o fato de que qualquer descuido pode colocar a empresa atrás das concorrentes.

A limpeza e a localização foram os atributos que receberam mais altos índices de desempenho, contrastando o fato de terem recebido, respectivamente, as segunda e terceira piores notas em relação à importância para o consumidor, atrás apenas da área de lazer. Isso indica que os hotéis, em alguns casos, estão concentrando a atenção em direções opostas às reais necessidades de seus clientes. No entanto, segundo Albrecht e Bradford (1992), é 
possível que um atributo que poderia ser importante para o cliente não esteja sendo valorizado, por algum motivo desconhecido. Nesse caso o problema provavelmente estará associado à ausência de políticas eficazes na comunicação dos benefícios relacionados ao bom desempenho do serviço/produto oferecido. Por exemplo, se o consumidor for informado de forma apropriada sobre os prejuízos que a má higienização provoca, ou a respeito da conveniência que a localização lhe proporcionará, ele possivelmente passará a estimar tais atributos e considerá-los ao escolher um local para se hospedar. Ainda tratando dessas variáveis, muitos estudos comparando a importância conferida a atributos de hotéis por turistas de negócios e turistas de lazer indicam que, enquanto os primeiros valorizam a limpeza e a localização, os de lazer atribuem altas notas à segurança, cortesia no atendimento e bons preços. (CLOW; GARRETSON; KURTZ, 1994; LEWIS, 1985; MARSHALL, 1993; PARASURAMAN et. al., 1988)

Atkinson (1988) definiu que, na escolha de um hotel, a limpeza dos alojamentos, seguida pela segurança, acomodação compatível com preços cobrados, cortesia e atendimento, são os atributos mais importantes para os hóspedes. Os resultados encontrados na presente pesquisa, contudo, no que diz respeito ao atributo limpeza, se revelam contrários. Outra pesquisa conflitante com esse estudo foi desenvolvida por Rivers, Toh e Alaoui (1991), os quais destacaram em seus resultados a conveniência da localização e os serviços gerais como fatores mais valorizados pelos clientes. Essas contradições possivelmente se devem, entre outros fatores, ao fato de as pesquisas não terem sido realizadas no Brasil, pois os agentes culturais exercem uma ampla e profunda influência no comportamento do consumidor (KOTLER, 2007), refletindo, assim, no grau de importância conferido às variáveis.

A área de lazer, por sua vez, foi constatada como uma variável muito pouco valorizada pelos clientes da rede hoteleira de Campo Grande e, conseqüentemente, seu desempenho não implica grande relevância para essas empresas. Esse atributo poderia ser utilizado como fator de diferenciação dos concorrentes, porém sua extrema desvalorização torna os investimentos desnecessários, sendo mais proveitoso concentrá-los em atributos do primeiro quadrante.

A agilidade e eficiência dos funcionários, bem como a segurança, foram avaliadas como muito importantes, mas não tiveram resultados totalmente satisfatórios. Agilidade e eficiência apresentaram desempenho bom, mas frente a sua extrema valorização, é necessário que esse 
desempenho seja aumentado para ótimo. Além de investir em novas tecnologias, como criação de terminais multimídia, desenvolvimento de novos sistemas de check-in e check-out e operações através de centrais de atendimento, os hotéis podem aperfeiçoar as habilidades do quadro de colaboradores por meio de treinamentos e recompensas aos bons funcionários.

Já a segurança foi o atributo com maior disparidade entre qualidade desejada e qualidade percebida. De acordo com Albrecht e Bradford (1992), para obter vantagem competitiva na gestão de serviços é necessário que a empresa compreenda os valores predominantes e como eles influenciam as atitudes dos clientes. Os hotéis de Campo Grande revelaram-se ineficientes na tarefa de perceber o valor desse atributo para seus clientes e transmitir a eles a segurança necessária. As providências que podem ser tomadas para melhorar a imagem dos hotéis incluem implantação de sistemas de vídeos, controle de acesso, renovação de equipamentos, utilização de detectores de incêndio e inclusão de um gestor de segurança na equipe de funcionários ou terceirização da segurança, entre outros.

Outro aspecto constatado como carente de maior cautela foi o serviço de alimentação dos hotéis. O atributo restaurante teve sua importância acima da média, no entanto foi o que apresentou o pior desempenho, não apenas na análise geral, mas em todas as categorias estudadas.

Por fim, a cortesia dos funcionários é merecedora de ênfase em relação às demais variáveis, pois foi a responsável pelos maiores níveis de satisfação. É um atributo extremamente valorizado e com desempenho muito bom, ocupando assim o lugar de destaque nas qualidades encontradas na rede hoteleira do município de Campo Grande.

Os resultados gerais corroboraram os encontrados por Zeithaml et al. (1990), que listaram as dimensões em ordem de importância relativa da seguinte forma: confiabilidade, atendimento, segurança, empatia e tangibilidade. Embora nesse estudo cada dimensão tenha recebido um número diferente de questões relacionadas a elas e, portanto, mensurar a ordem exata seja muito complexo, fica evidente que os aspectos referentes à confiabilidade, ao atendimento e à segurança apresentaram níveis de importância muito acima dos demais atributos, para todas as categorias de hotéis. Portanto, os critérios finais de serviços que os hotéis de Campo Grande, de modo geral, devem assumir são: segurança, cortesia dos funcionários e atributos referentes à dimensão confiabilidade. 
Segue abaixo as matrizes referentes a cada categoria de hotel:
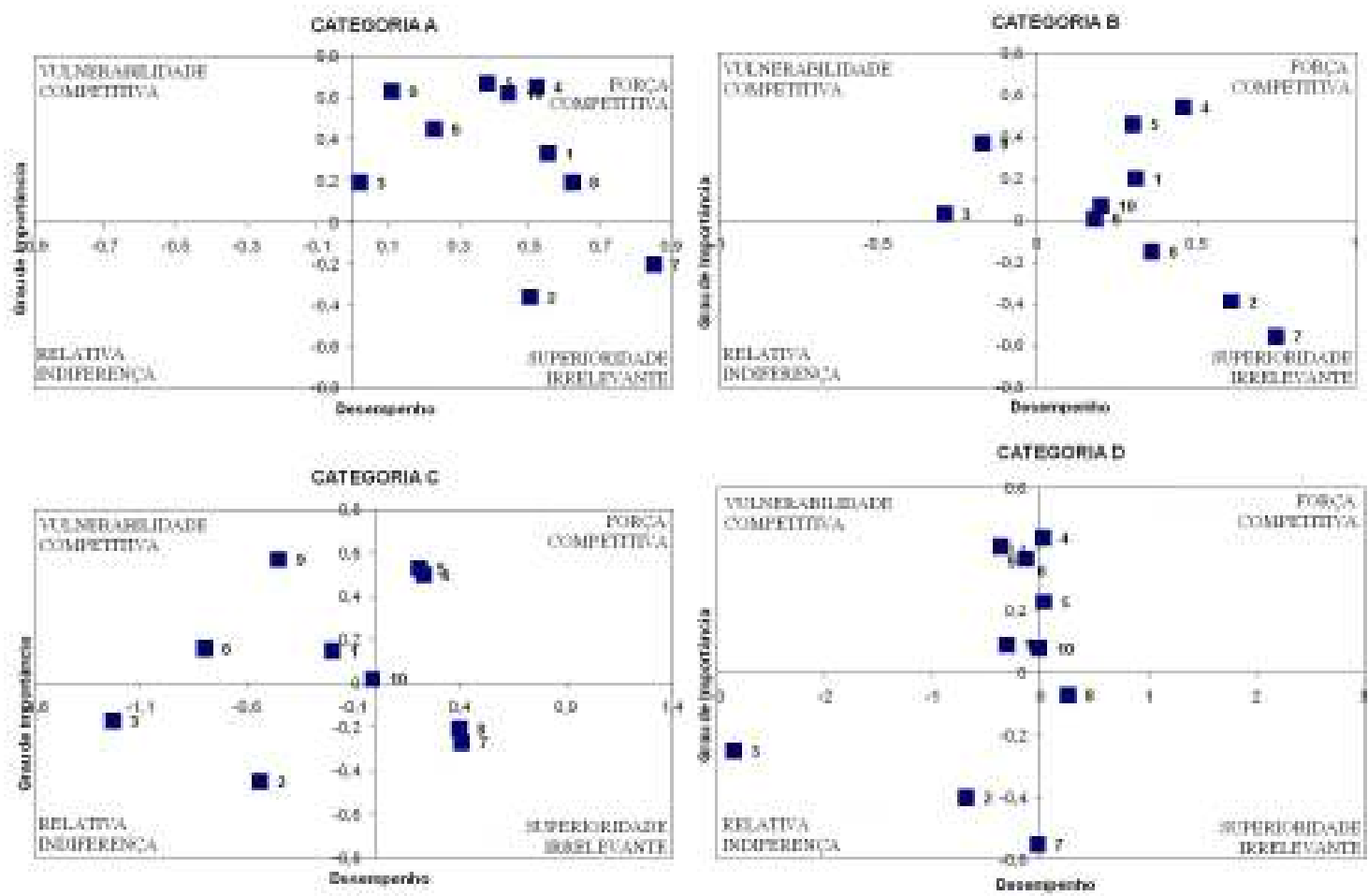

Figura 3 - Matriz de atributos dos hotéis por categoria - (1) acomodação; (2) área de lazer; (3) restaurante; (4) cortesia dos funcionários; (5) agilidade e eficiência dos funcionários; (6) conhecimento dos funcionários; (7) limpeza; (8) localização do hotel; (9) segurança; (10) atenção personalizada.

Fonte: Elaborado pelos autores

\subsection{Resultados para os hotéis pertencentes à categoria $\mathrm{A}$}

Nos hotéis classificados na pesquisa como pertencentes à categoria $\mathrm{A}$, não foram encontradas variáveis que apresentam vulnerabilidade competitiva, nem relativa indiferença, como pode ser observado na Figura 3. Nessa classe de hotéis a grande maioria das variáveis concentrouse na segunda área, revelando um alto grau de satisfação dos consumidores, uma vez que tanto a importância como a performance são altas. Cortesia, agilidade e eficiência, atenção personalizada e segurança apareceram no topo dos critérios que determinam a escolha do hotel pelos consumidores dessa categoria, já que, como pode ser visto na Figura 3, ocupam o nível mais elevado no grau de importância. Apesar de todos terem apresentado desempenho 
avaliado como bom por mais de 50\% dos entrevistados, esses atributos não se encontram no topo dos valorizados pelos hotéis. As variáveis com mais alto desempenho foram limpeza e localização, que não agregam valor significativo, de acordo com o estudo. Esse fator revela que os hotéis pertencentes à classe A poderiam melhor alocar seus recursos e, assim, atrair seu público e satisfazê-lo em suas reais necessidades.

As variáveis com menor importância do ponto de vista dos consumidores são área de lazer e limpeza. Nesse caso, os hotéis devem criar estratégias para que os consumidores percebam os atributos em questão como geradores de vantagens, ou, se não existir força suficiente para que a importância atribuída seja elevada, a empresa pode investir menos esforços e recursos, já que tais investimentos não surtem efeito relevante.

Os critérios finais de serviço nesse caso poderiam ser o conhecimento dos funcionários e a segurança oferecida aos hóspedes. São variáveis que, apresentando desempenhos excelentes, podem trazer à empresa diferenciação de mercado.

\subsection{Resultados para os hotéis pertencentes à categoria $B$}

A matriz dos hotéis da categoria B apresenta variáveis segurança e restaurante no primeiro quadrante. Ou seja, essas variáveis foram classificadas pelos consumidores como importantes, no entanto, o desempenho alcançado foi baixo. Além disso, área de lazer, limpeza e localização foram classificados como irrelevantes Esse resultado mostra que há fatores importantes sendo negligenciados, como também atenta para o fato de que os hotéis estão concentrando investimentos em áreas que não geram vantagem competitiva.

Para o público da rede hoteleira considerada como classe $\mathrm{B}$, as variáveis de maior valor, além da segurança, que obteve desempenho ruim, são cortesia, agilidade e eficiência dos funcionários, seguidas por acomodações e atenção personalizada. Embora tenham apresentado bons resultados, nenhuma delas foi classificada como tendo ótimo desempenho, o que implica na necessidade de aperfeiçoamento das estratégias e melhorias em relação aos serviços prestados, principalmente no que se refere ao quadro de funcionários. Algumas das providências que podem ser tomadas pelas empresas são: melhorar o processo de seleção de funcionários, oferecer treinamentos, aquisição de equipamentos e sistemas com tecnologias que permitam maior eficiência e agilidade e investir na estrutura das acomodações, entre outras. 


\subsection{Resultados para os hotéis pertencentes à categoria $C$}

Os atributos mais importantes para os clientes dessa classe são a segurança, a agilidade e eficiência dos funcionários e a cortesia dos mesmos. Os dois últimos apresentaram desempenho razoavelmente bom, ainda que seja recomendável às organizações que busquem aumentar o desempenho de tais variáveis, uma vez que a decisão sobre a hospedagem depende essencialmente delas.

A segurança, contudo, que está no topo de estima, foi avaliada como ruim. Fora a categoria de hotéis $\mathrm{A}$, todas as demais obtiveram resultados insatisfatórios em relação a esse atributo, o que indica uma possível negligência da rede hoteleira como um todo sobre a segurança oferecida aos hóspedes. Além dela, apresentação das acomodações e conhecimento dos funcionários foram atributos tidos como importantes para os clientes que também obtiveram avaliações negativas.

A atenção personalizada foi a única variável localizada na zona cinzenta, já que a ela foram atribuídos níveis de importância e desempenho médios. Foi constatado ainda que as variáveis área de lazer e restaurante têm pouco valor para esses consumidores, logo o baixo desempenho não é um fator relevante. A limpeza e localização do hotel também são atributos nos quais as empresas da classe C não precisam concentrar intensos esforços, uma vez que essas variáveis não agregam grande valor aos hotéis e já possuem uma performance superior ao seu grau de importância.

\subsection{Resultados para os hotéis pertencentes à categoria $D$}

$\mathrm{Na}$ categoria $\mathrm{D}$, a grande maioria dos atributos encontra-se entre o primeiro e segundo quadrantes. A apresentação das acomodações, conhecimento dos funcionários e a segurança estão localizadas no primeiro quadrante, porém ainda bastante próximas do desempenho mediano. De acordo com esses resultados, fica evidente que esses devem ser os critérios finais de serviço para esta categoria de hotel.

Apesar do péssimo desempenho, a área de lazer e o restaurante foram atributos pouco importantes de acordo com os consumidores, o que implica em uma relativa indiferença. A limpeza do hotel apresentou desempenho satisfatório, pois mesmo tendo sido avaliado como 
razoável, como foi considerado o fator menos significante, sua excelência torna-se dispensável. A localização foi a única variável com superioridade irrelevante.

\subsection{Comparação entre as categorias de hotéis}

A Figura 4 exibe a diferença entre as médias de expectativa e de desempenho dos hotéis de categoria A, B, C e D, isto é, quanto mais próximas de zero as médias estiverem, maior será a relação entre expectativas e desempenho. O ponto zero é onde expectativas e desempenho se igualam.

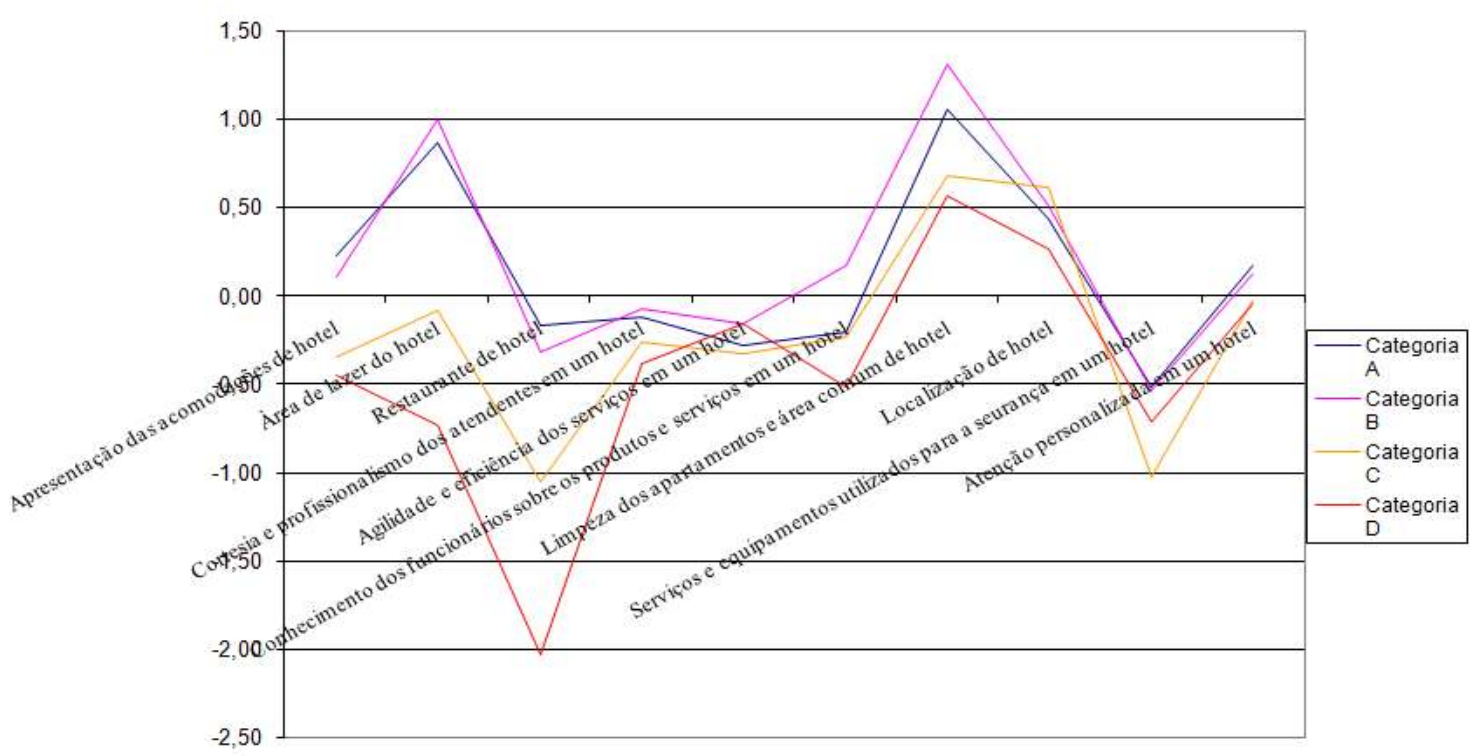

Figura 4 - Diferença entre as médias de expectativa e de desempenho dos hotéis

Fonte: Elaborado pelos autores

Como pode ser visto na figura, os hotéis das categorias A e B são os que melhor atendem seus consumidores. Para ambos a cortesia dos funcionários é o atributo que possui menor divergência entre expectativas e desempenho. Nos hotéis de categoria A também há proximidade das médias de restaurante e atenção personalizada em relação ao ponto zero, sendo que nos de classe B a apresentação das acomodações ocupa o lugar dos restaurantes. A área de lazer e a limpeza do hotel são as variáveis mais distantes. Para os hotéis C e D é a 
atenção personalizada o atributo com maior semelhança entre expectativas e desempenho, enquanto restaurante e segurança são os mais discrepantes.

Com base na análise das figuras que apresentam os resultados de cada categoria de hotel e da figura de comparação entre todas elas, pode-se observar que os hotéis da categoria A são os únicos que satisfazem seus clientes em todos os atributos pesquisados, uma vez que para esses consumidores o desempenho está igual ou acima de suas expectativas. Em seguida estão os hotéis $\mathrm{B}$ e $\mathrm{C}$, respectivamente, e, por último, classificados como os que menos satisfazem seu público, encontram-se hotéis de classe D.

\section{Considerações Finais}

Esse trabalho teve como objetivo avaliar a satisfação dos consumidores da rede hoteleira de Campo Grande, através da mensuração das expectativas dos serviços prestados e do desempenho percebido pelos clientes. Para tanto, foi feito um estudo quantitativo junto a 407 usuários de hotéis de Campo Grande-MS.

A análise do perfil dos consumidores, através da matriz de atributos, permitiu identificar não apenas o nível de satisfação dos consumidores, mas também as variáveis que devem receber maior atenção e investimentos por parte dos hotéis, para que dessa forma possam elaborar estratégias bem direcionadas aos clientes, criando assim vantagem competitiva e condições para concorrer em um mercado onde a disputa está cada vez mais acirrada.

Com base na problemática do estudo e de acordo com os resultados encontrados por meio da análise da pesquisa realizada, percebe-se que a rede hoteleira de Campo Grande, de um modo geral, tem satisfeito seus clientes na maior parte das dimensões avaliadas. Os únicos atributos que apresentaram desempenho bastante diferente do esperado, de forma negativa, foram a segurança e os serviços de alimentação. Para os consumidores, as instalações físicas dos hotéis, bem como o atendimento, a empatia e a confiabilidade, agregam serviços e produtos que variam de bons a ótimos. Contudo, para adquirir excelência e ganhar participação no mercado, os hotéis devem continuar capacitando os funcionários, buscando conhecer seu público com mais profundidade e investindo constantemente em novas técnicas de gestão que possibilitem atendê-los da melhor forma. 
Eficiência, agilidade, cortesia e conhecimento são atributos que requerem atenção especial. Os hotéis deveriam investir mais em treinamento e capacitação de seu quadro de colaboradores, além de promover maior envolvimento de todos os funcionários para manter o bom nível da qualidade da prestação de serviços.

Os atributos determinantes para a satisfação dos consumidores foram definidos como sendo a segurança oferecida pelas instalações, equipamentos e funcionários aos clientes e a cortesia, agilidade e eficiência com que são atendidos.

Quanto às limitações deste estudo, pode-se destacar a precariedade de informações sobre o setor hoteleiro no município de Campo Grande e, à restrição dos resultados obtidos apenas ao local pesquisado, o levantamento de dados ter sido feito de maneira transversal única, restringindo assim os resultados a um período no tempo, além da pequena quantidade de variáveis mensuradas por limitar as possíveis discussões.

Esse trabalho apresenta implicações organizacionais ao trazer uma análise sobre a importância de conhecer as crenças, valores e necessidades dos clientes a fim de criar estratégias que garantam um posicionamento de vantagem competitiva, gerando aumento de lucro e participação de mercado. Trouxe, ainda, a possibilidade para a rede hoteleira de Campo Grande de distinguir os atributos valorizados pelos seus consumidores, bem como seus pontos fortes e fracos e o nível de desempenho que tem alcançado do ponto de vista do público-alvo. A academia terá um arcabouço maior de conhecimento sobre o comportamento dos consumidores/usuários de hotéis, contribuindo para futuros estudos.

\section{Referências}

ABIH- Associação Brasileira da Indústria de Hotéis. 2008. Disponível em: <www.abih.com.br> Acesso em: 18 jun. 2009.

ALBRECHT, K.; BRADFORD L. J. Serviços com qualidade: a vantagem competitiva. São Paulo: Makron Books do Brasil, 1992.

ANDERSON, R. E. Consumer satisfaction: the effect of disconfirmed expectancy on perceived product performance. Journal of Marketing Research, v.10, n.2, p. 38-44, 1973.

ANDERSON, W. E.; MITTAL, V. Strengthening the satisfaction-profit chain. Journal of Service Research, vol.3, n.2, 107-120, 2000.

ATKINSON, A. Answering the eternal question: What does the customer want? The Cornell Hotel and Restaurant Administration Quarterly, v.29, n.2, p. 12-14, 1988. 
BARSKY, J.; NASH, L. Customer satisfaction: applying concepts to industry-wide measures. Cornell Hotel and Restaurant Administration Quaterly, v. 44, n.5-6, p.173-183, 2003.

BERRY, L. L.; PARASURAMAN A. Serviços de Marketing: competindo pela qualidade. São Paulo: Maltese, 1995.

BRANCO, G. M.; RIBEIRO, J. L. D.; TINOCO, M. A. C. Determinantes da satisfação e atributos da qualidade em serviços de hotelaria. Produção, v. 20, n. 4, p. 576-588, out./dez. 2010.

CARVALHO, S. et al. Situação da gestão de resíduos no setor de hotelaria. Revista Nordestina de Ecoturismo, Aracaju, v. 2, n. 2, out. 2009.

CARDOZO, R. N. An experimental study of customer effort, expectation and satisfaction. Journal of Marketing Research, v. 3, p. 244-249, 1965.

CHURCHILL, G. A.; SURPRENANT, C.; An Investigation Into the Determinants of Customer Satisfaction. Journal of Marketing Research, v. XIX, n. 4, p. 491-504, 1982.

CLOW, K. E., GARRETSON, J. A.; KURTZ, D. L. An exploratory study into the purchase decision process used by leisure travellers in hotel selection. Journal of Hospitality \& Leisure Marketing, vol.2, n.4, 1994, p. 53-72.

EMBRATUR. Regulamento e matriz de classificação dos meios de hospedagem. 23 de abril de 2002. Disponível em:< http://www.embratur.gov.br> Acesso em: 24 jun. 2009.

EVRARD, Y. A Satisfação dos Consumidores: A situação das pesquisas. Working Paper, 1994.

GONZÁlES, M. O. A.; RAMOS, R. E. B.; AMORIM, C. G. Gestão da satisfação e da fidelidade do cliente na hotelaria: um estudo dos fatores que influenciam a satisfação e a fidelidade do turista internacional no Brasil. In: ENCONTRO NACIONAL DE ENGENHARIA DE PRODUÇÃO, 25., 2005, Porto Alegre. Anais... Porto Alegre: ENEGEP, 2005.

IBGE. Instituto Brasileiro de Geografia e Estatística. Economia do turismo: Uma perspectiva macroeconômica 2003-2007. Rio de Janeiro, 2010. Disponível em: $<$ www.dadosefatos.turismo.gov.br/export/sites/default/dadosefatos/outros_estudos/estudos_ibge/down loads_estudos_pesquisas_IBGE/Economia_do_Turismo_Uma_perspectiva_macroeconxmica_-_20032007_IBGE.pdf.> Acesso em: 26 jun 2011.

ISMAIL, A. Hospedagem Front Office e Governança. São Paulo: Pioneira Thomson Learning, 2004.

KOTLER, P.; ARMSTRONG, G. Princípios de Marketing. 12. ed, São Paulo: Pearson Prentice Hall, 2007.

LEWIS, R. C. Getting the most from marketing research [Part V]. Predicting hotel choice: The factors underlying perception. The Cornell Hotel and Restaurant Administration Quarterly, vol.26, n.3, p. 8296, 1985.

MACHADO, F. B. A Fidelização do consumidor no setor de serviços: uma análise comparativa entre setores de naturezas diferentes. Rio de Janeiro: PUC, 2001. Dissertação (Mestrado em Administração), PUC, Rio de Janeiro, 2001.

MALHOTRA, N. Pesquisa de Marketing: uma orientação aplicada. 3a ed. Porto Alegre: Bookman., 2001.

MARSHALL, A. Safety top guest's priority list; sell security as No. 1 amenity. Hotel and Motel Management, 1993.

OLIVER, R. L. Conceptualization and measurement of disconfirmation perceptions in the prediction of consumer satisfaction. Dissatisfaction and complaint behavior, 1980. 
OLIVER, R. L. Satisfaction - a behavioral perspective on consumer. Vanderbilt University/McGrawHill, 1997.

OLSHAVSKY, R. W.; MILLER, J. A. Consumer expectations, product performance, and perceived product quality. Journal of Marketing Research, v.9, p.19-21, 1972.

PARASURAMAN, A.; ZEITHAML, V. A.; BERRY, L. L. Reassessment of expectations as a compraison standard in measuring service quality: Implications for further research. Journal of Marketing, 58, 111-133, 1994.

PARASURAMAN, A., ZEITHAML, V. A.; BERRY, L. L. SERVQUAL: A multiple-item scale for measuring consumer perceptions of service quality. Journal of Retailing, vol.64, n.1, p. 12-37, 1988.

PROSÉRPIO, R. O avanço das redes hoteleiras internacionais no Brasil. São Paulo: Aleph, 2007.

RIVERS, M. J.; TOH, R. S.; ALAOUI, M. Frequent-stayer programs: The demographic, behavioural, and attitudinal characteristics of hotel steady sleepers. Journal of Travel Research, vol.30, n.2, p. 4145, 1991.

SPRENG, R. A.; MACKENZIE, S. B.; OLSHAVSKY, R.W. A Reexamination of the Determinants of Consumer Satisfaction. Journal of Marketing, n. 3, v. 6, p. 15-32, 1996.

TSE, D. K.; NOCOSIA, F. N.; WILTON, P. C. 1990. Consumer satisfaction as a process. Psychology and Marketing, 1990.

YI, Y. (1990). A critical review of consumer satisfaction. In V. Zeithaml (Ed.), Review of Marketing, American Marketing Association, v.4, p. 68-123, 1990.

ZEITHAML, V. A. et al. Delivering Quality Services: balancing customer perceptions and expectations. New York: The Free Press, 1990.

Recebido em: 19/09/2011

Aprovado em: 25/11/2011 (1 ${ }^{\mathrm{a}}$ versão) 02/12/2011 (2 $2^{\mathrm{a}}$ versão) 